\title{
SIMULATION OF THE BETATRON MAGNETIC FIELD AT THE ELECTRON BEAM DISPLACEMENT IN COMSOL MULTIPHYSICS
}

\author{
Andrey Kolomeytsev, Vladimir Smolyanskiy* and Igor Stepanov \\ National Research Tomsk Polytechnic University, 634028, Tomsk, Russia
}

\begin{abstract}
This article describes the betatron magnetic field simulation in Comsol Multiphysics software package. The model makes it possible to evaluate the betatron focusing properties at the moment of electron displacement onto the target. The simulation results coincide with the experiment described earlier. The study shows the vertical size of the target affects the focal spot size. However, a target size decrease leads to low output dose, since not all electrons reach it. Therefore, the vertical size of the target should be greater than or equal to the vertical dimension of the accelerated beam.
\end{abstract}

\section{Introduction}

Betatrons have found wide application in terms of defectoscopy, medicine and X-ray industrial tomography. The main difference between betatrons and other radiation sources is a narrow radiation pattern of the beam and, consequently, a lower radiation hazard in non-stationary applications, portability and high energy of the accelerated beam. In particular, this makes it possible to introduce betatrons into modern tomographic complexes for the testing of large-sized objects, including containers and vehicles. Betatrons are also used in electronic equipment radiation testing with the aim of predicting the service life in space conditions [1-10]. However, such systems have high requirements to the magnetic field characteristics to ensure radiation stability. Improving the betatron characteristics is a continuous process. This is due to the ever-growing demands of the radiation source market.

Close to $10^{12}$ electrons are injected into betatron in one acceleration cycle. Only less than $1 \%$ reach the target and generate bremsstrahlung. There are many experimentally verified reasons for such an assertion. Among them may be the following:

- Insufficient energy of beam displacement.

- Low density of electron space charge.

- Low synchronization accuracy between the beam displacement and the accelerating magnetic flux.

- Insufficient stability of voltage pulse parameters.

- Non-optimal geometry and mutual arrangement of electrodes.

\footnotetext{
*Corresponding author: aak65@tpu.ru
} 
Each factor influence evaluation entails a large number of complex experiments. However, it can be avoided by using computer-aided engineering systems. Computer simulation makes possible to more deeply study the internal processes in the betatron magnetic field during operation and save considerable time and resources.

This paper is devoted to the study of the betatron magnetic field during the electron beam displacement Comsol Multiphysics software package is chosen as a simulation environment.

\section{Materials and methods}

The betatron field has axial symmetry. The electron moves in the magnetic field along the potential minimum line. Its orbit has the form of a circle. This trajectory is called the equilibrium orbit. In this orbit, the axial forces hold the electron in the median plane and the radial component of the Lorentz force is compensated by the centrifugal forces. Such an orbit exists if the induction of the magnetic field $\mathrm{B}_{0}$ at the points of this orbit at any time instant is 2 times less than the average induction of the magnetic field $B_{a v}$ inside the orbit.

When accelerated electrons reach a predetermined energy, they should either be directed at the target to form bremsstrahlung or be withdrawn from the vacuum chamber in the form of a direct electron beam. In either case, the electrons must be displaced from the equilibrium orbit. For this purpose, special displacement schemes are used in inductive cyclic accelerators. The most widely used method of electron displacement from an equilibrium orbit to a target is to change the magnetic field distribution in the accelerator interpolar space. It is achieved by passing current through special displacement coils.

a

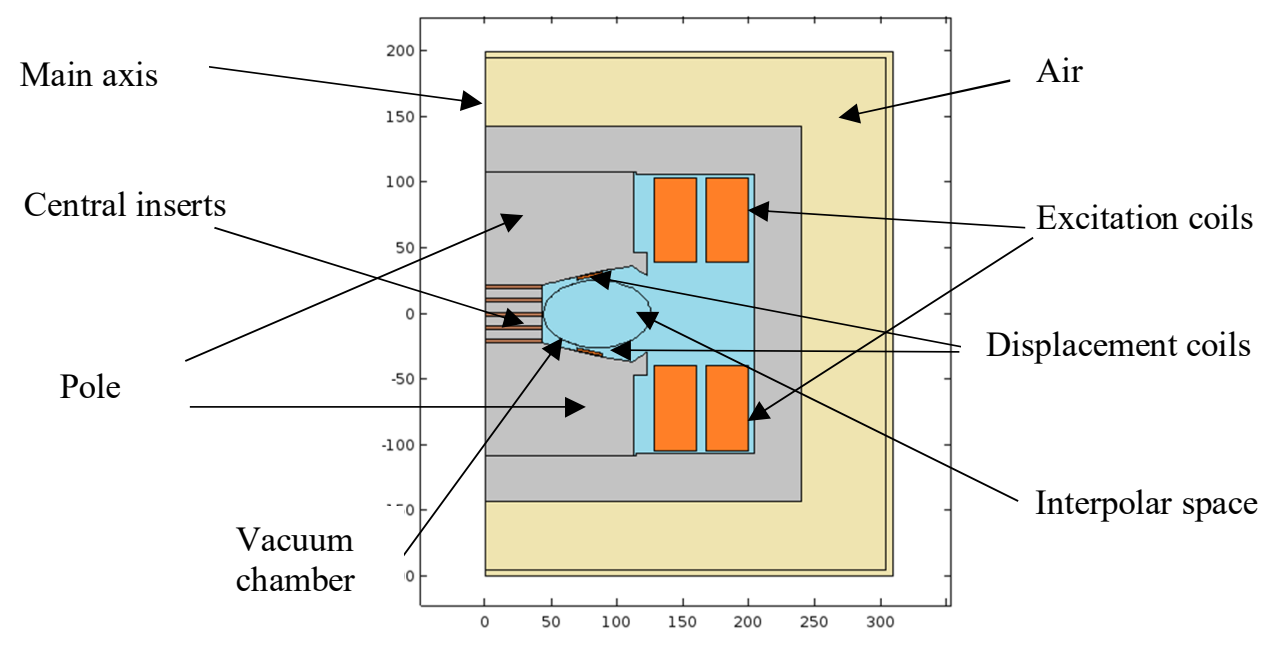

Fig. 1. Magnetic circuit elements.

The paper considers a magnetic field during the electron beam displacement in a smallsize betatron MIB-10 with an output electron energy of $10 \mathrm{MeV}$. Figure 1 shows the magnetic system main elements. The operating frequency is $150 \mathrm{~Hz}$. The injector is installed inside the vacuum chamber. Because of thermionic emission, an electron flux equivalent to the current strength of $0.6-0.8 \mathrm{~A}$ is pulled out from the cathode surface. Under the influence of the potential difference between the anode and the cathode, the electrons are accelerated to energy of $40 \mathrm{keV}$ and fall into the interpolar space. In the interpolar space, electrons tend to an equilibrium orbit and are uniformly distributed along it under the action of the Coulomb force. Further, the electrons are accelerated in the 
growing magnetic field. At the end of the acceleration cycle, a current is applied to the displacement coils, which distorts the magnetic field in such a way that the equilibrium orbit radius begins to increase until the electron beam reaches the target established at the anode of the injector. The injector with the target is located at a radius of $110 \mathrm{~mm}$ relative to the betatron main axis.

To increase the equilibrium orbit radius and the electron beam displacement to the external target, it is necessary to reduce the field in the orbit region. The change magnitude in the $d_{0}$ field required to change the radius of the orbit by a value of $d R=R_{m}-R_{0}$ is given by:

$$
\mathrm{dB}_{0}=\mathrm{B}_{0}\left(\frac{\mathrm{R}_{0}}{\mathrm{R}_{\mathrm{m}}}-\left(\frac{\mathrm{R}_{0}}{\mathrm{R}_{\mathrm{m}}}\right)^{\mathrm{n}}\right)
$$

Loop coil that create a displacing magnetic field are located above and below the equilibrium orbit between the vacuum chamber walls and the surfaces of the betatron poles. The most common scheme for beam displacement on a target is an oscillating (discharge) circuit with a storage capacity of $\mathrm{C}$. Neglecting the active energy losses in the discharge circuit, the amplitude current value can be determined as:

$$
\operatorname{Im}=\omega_{0} \cdot \mathrm{C} \cdot \mathrm{U}_{\mathrm{c}}
$$

where $\omega_{0}=\frac{\pi}{t_{c}}-$ is the natural oscillation frequency of the discharge current, $t_{c}-$ is the displacement pulse duration, $U_{c}-$ is the capacitor voltage, $V$ [11].

The discharge efficiency drops sharply with an insufficient displacement current. This is easily explained by the fact that the electron beam does not reach the target and bremsstrahlung does not arise. In addition, the current change rate affects on the focal spot dimensions. A more detailed study of this effect will allow creating sources with a variable focal spot size that will not depend on the accelerated electron energy.

The electron kinetic energy is always consistent with the magnetic field induction. In other words, there is a relationship between magnetic induction on an equilibrium orbit and the electron energy. This relationship can be described by an empirical formula:

$$
\left(\mathrm{W}\left(\mathrm{W}+2 \mathrm{E}_{0}\right)^{\frac{1}{2}}=300 \mathrm{~B}_{0} \mathrm{R}_{0}\right.
$$

where $R_{0}$ - is the equilibrium orbit radius, $\mathrm{m} ; B_{0}-$ is the magnetic induction in the equilibrium orbit, $T ; E_{0}$ - is the electron rest energy.

Knowing the equilibrium radius, we can calculate the induction value in the equilibrium orbit at the time when the electron energy reaches $10 \mathrm{MeV}$.

$$
\mathrm{B}_{0}=\frac{\left(\mathrm{W}\left(\mathrm{W}+2 \mathrm{E}_{0}\right)^{\frac{1}{2}}\right.}{300 \mathrm{R}_{0}}
$$

All the values obtained give us enough information to create and calculate the betatron computer model.

\section{Results and discussion}

The simulation results show how the induction of the magnetic field is distributed in the magnetic circuit. As shown in Figure 2, the largest induction in the central block. A further increase in the current in the inductive coils will lead to saturation of the magnetic inserts in the central block. As a result, the average induction at $\mathrm{R}<\mathrm{R}_{0}$ will grow slower than the induction at $\mathrm{R}>\mathrm{R}_{0}$, which will lead to decrease radius. 


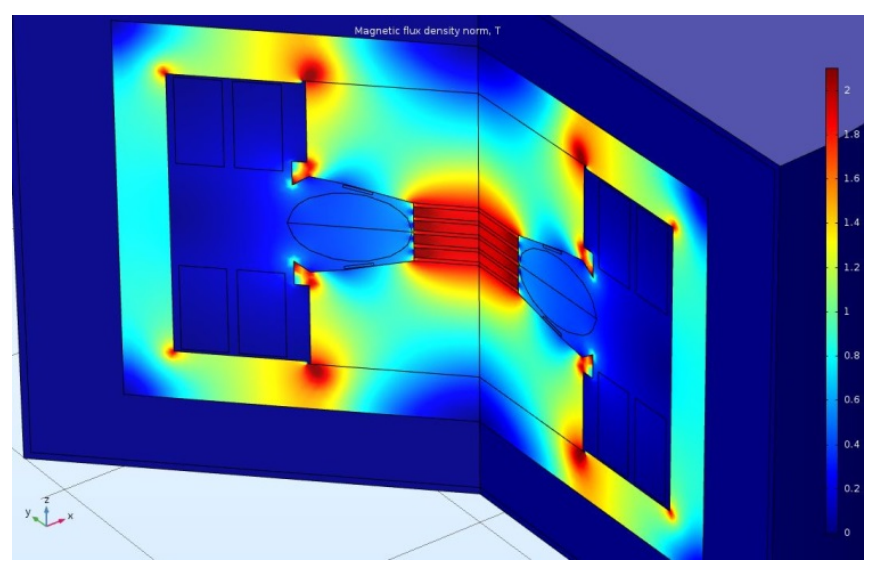

Fig. 2. Magnetic flux density before displacement start.

The further current increase in the excitation coils is undesirable, since the target is at a larger radius than the equilibrium orbit. This means that the electrons have acquired the maximum possible energy from the accelerating field and it is time to displace them onto the target. Let us consider the potential function at this time point.

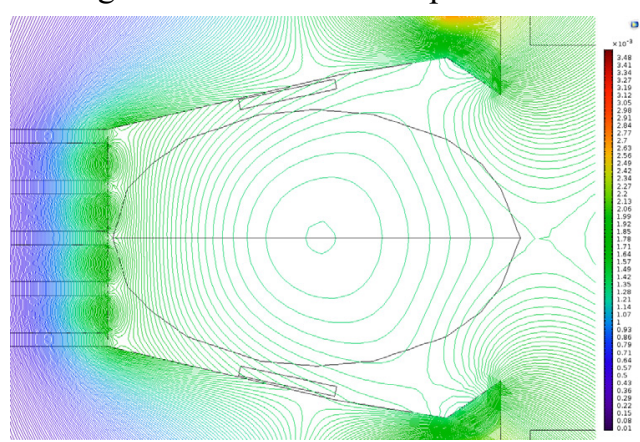

(a) $\mathrm{I}=0$

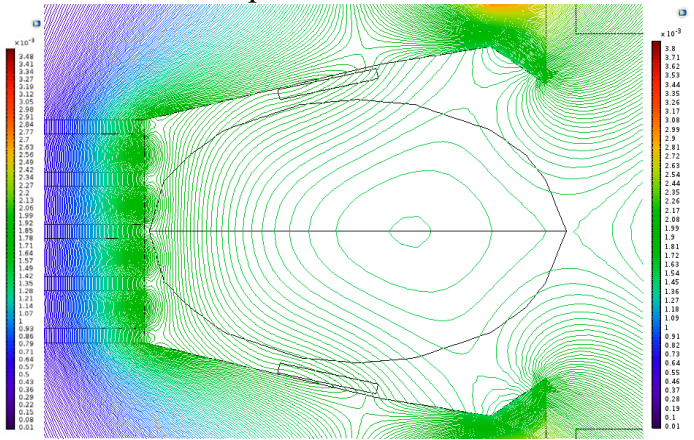

(b) $\mathrm{I}=0.5 \mathrm{Im}$

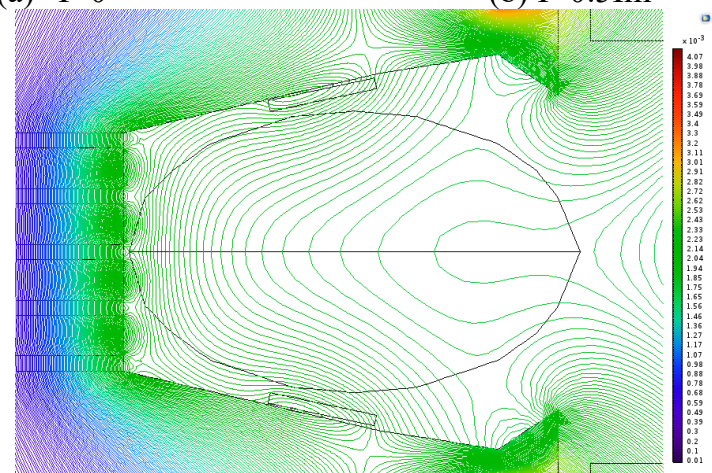

(c) $\mathrm{I}=\mathrm{Im}$

Fig. 3. Potential function while displacement.

As can be seen from Figure $3 a$, the isolines of the potential function inside the accelerator chamber are concentrated around the equilibrium orbit. Experiments show that the diameter of the electron beam is $2-3 \mathrm{~mm}$. This diameter is obtained when the balance of centrifugal and magnetic forces and the forces of the Coulomb interaction. A current 
increase in the displacement coils (Figure 3b,c) leads to an expansion of the equilibrium orbit. In the wake of this, the electron beam orbit expands. This is because the magnetic flux at $\mathrm{R}<\mathrm{R}_{0}$ increases, and at $\mathrm{R}>\mathrm{R}_{0}$ it remains unchanged (Figure 4 ). The change in the ratio of the average induction and induction on the radius leads to an expansion of the orbit.

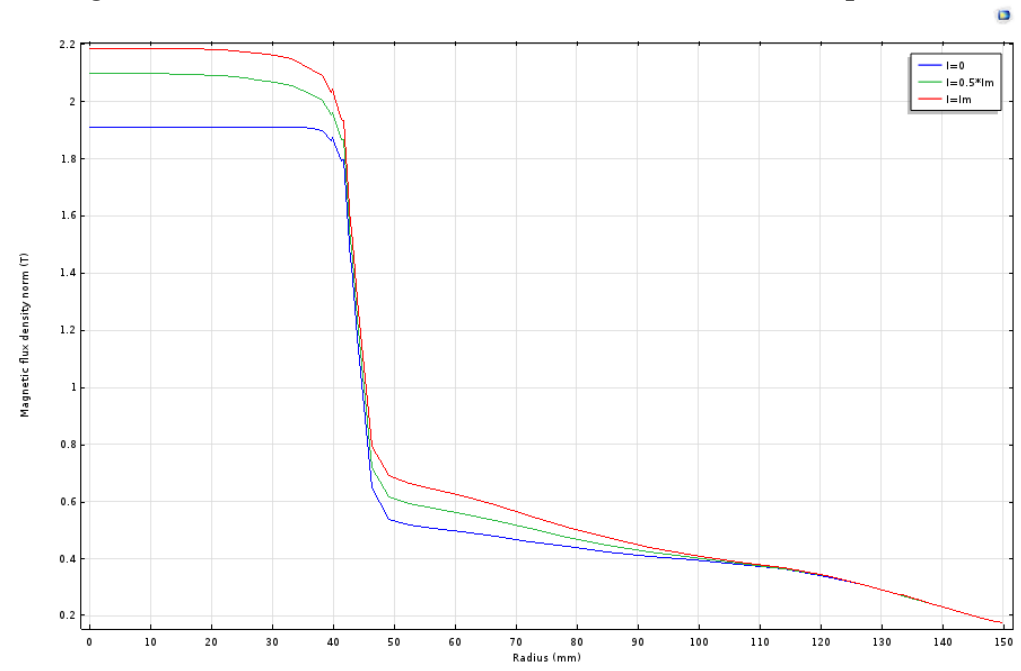

Fig. 4. Magnetic flux density against radius at median plane.

Ability to focus and hold the electron beam at all stages of the work is crucial for betatron operation. The potential function or rather the potential well depth also determines the betatron focusing properties. The simulation results show that the vertical focusing is preserved (Figure 5.) with the equilibrium orbit displacement. This means that there is no precondition to increase the vertical beam size during the electron beam displacement to the target.

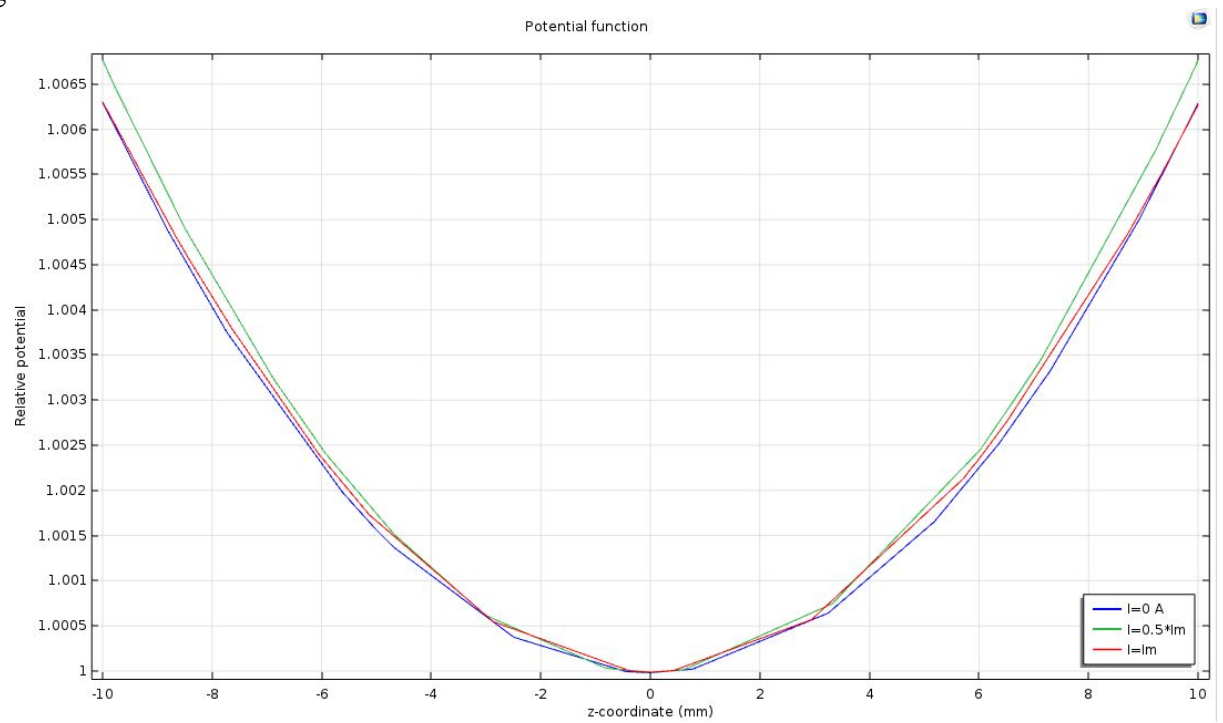

Fig. 5. The potential function against axial coordinate at the potential minimum.

Research [12] shows that the target vertical size directly affects the size of the focal spot. However, a decrease in the target leads to a decrease in the output dose, since not all 
electrons reach the target. Therefore, the vertical size of the target must be greater than or equal to the vertical dimension of the accelerated beam.

The graph of the radial focusing is shown in Figure 6. As the displacement current increases, the potential barrier at $\mathrm{R}>\mathrm{R}_{0}$ decreases. The radial oscillations of the electrons increase. Too rapid increase in the displacement current will cause electrons fly past the target and fall on the anode box. The output dose will be significantly reduced.

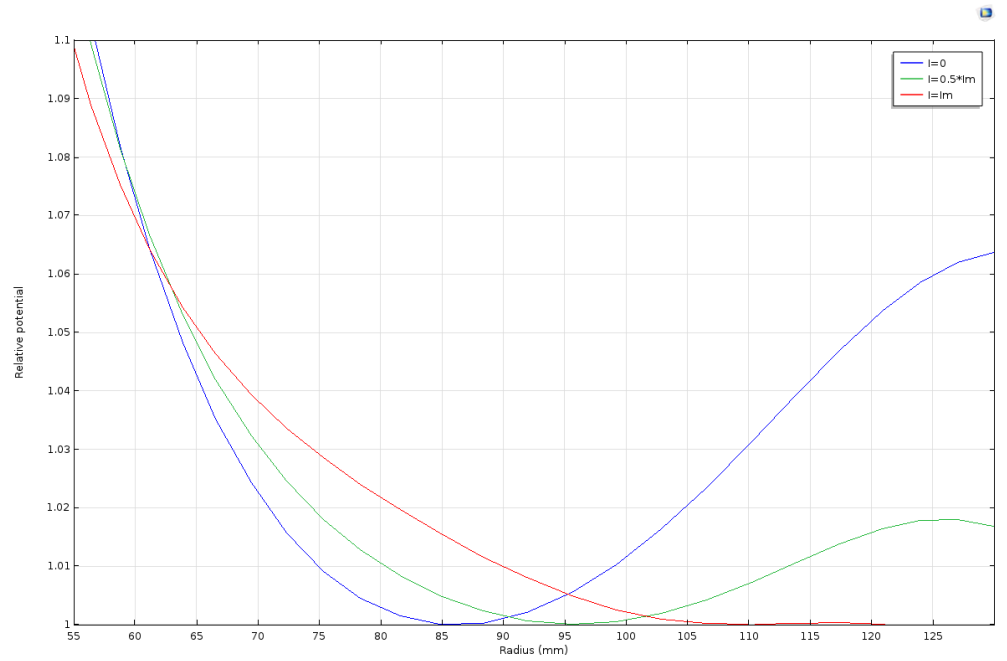

Fig. 6. The radial component of the potential function at median plane.

The simulation results allow us to estimate the qualitative and quantitative characteristics of the betatron magnetic field in the electron beam displacement process. These data will be used to optimize and find new solutions in improving the betatron efficiency.

\section{Conclusion}

In this paper, a betatron computer model is presented. This model describes the betatron magnetic field at the moment of the displacement of electrons from the equilibrium orbit. Increasing the beam displacement efficiency will allow us to achieve the characteristics of sources based on linear accelerators.

The experiments validate the simulation results. Betatron operation simulate helps to better understand the ongoing physical processes. This simulation allows us to quickly find the optimal design solution when developing new accelerator models.

It should be noted that despite the low prevalence of betatrons with respect to linear accelerators, they have a much greater potential for development. Achievement of the set goals will allow expanding the device application area of this device.

\section{Acknowledgments}

The research was supported by the Russian Science Foundation (Grant \#17-19-01217).

\section{References}

[1] L. De. Chiffre, S. Carmignato, J-P. Kruth, R. Schmitt and A. Weckenmann, CIRP An - MT 63, 655 (2014) 
[2] N. Schuhmann and H. Okruch, DGZfP Proc, BB67 (1999)

[3] V. Smolyanskiy, M. Rychkov and V. Borikov, MATEC WoC 102, 01033 (2017)

[4] L. Bonaccorsi, F. Garescì, F. Giacobbe, F. Freni, F. Mantineo, R. Montanini and A. Sili 105, 33 (2013)

[5] A. du Plessis, S. G le Roux and A. Guelpa, CS in NDT\&Ev 6, 17 (2016)

[6] N. Reims, T. Schoen, M. Boehnel, F. Sukowski and M. Firsching, Proc of SPIE 921209, (2014)

[7] W. Holub and U. Hassler, NDT in Canada, (2013)

[8] E .Vaynberg et al., 16th World Conf. NDT. 1, (2004)

[9] G. Herman, Fundamentals of Computerized Tomography. (The Graduate Center, CUNY, New York, 2009)

[10] M. Stein, V. Kasyanov, V. Chaklov, J. Macleod, P. Marjoribanks and S. Hubbard, WCNDT (2004)

[11] V. Moskalev and V. Chaklov, Betatron: monography (TPU, Tomsk, 2009)

[12] V. Kasyanov et al., Instr. \& Exp. Tec's. 41, 41 (1998) 